\title{
Desenvolvimento da Versão Reduzida do Inventário de Jesness - Revisado Brasileiro
}

\author{
Rafaelle C. S. Costa, André Vilela Komatsu, Lais Sette Galinari, Marina Rezende Bazon ${ }^{1}$ \\ Universidade de São Paulo, Ribeirão Preto-SP, Brasil
}

\section{RESUMO}

O objetivo do estudo foi selecionar itens para composição da versão reduzida do Inventário de Jesness - Revisado brasileiro, instrumento específico de avaliação psicológica de adolescentes infratores. Foram utilizados dados de 954 adolescentes da população, com idades entre 11 e 18 anos, do sexo masculino. Realizaram-se análises do Modelo Exploratório de Teoria de Resposta ao Item (EMIRT) e, em seguida, do Modelo Confirmatório de Teoria de Resposta ao Item (CMIRT), para cada escala do instrumento, separadamente. Na etapa do EMIRT foram excluídos itens com $\alpha$ inaceitável; na do CMIRT mantidos apenas itens com $\alpha$ moderado ou alto. Denotou-se existência de subdimensões nas escalas, sendo que alguns itens se mantiveram em mais de uma escala. Chegou-se assim a uma versão com 86 itens. As estratégias adotadas permitiram selecionar itens com maior capacidade discriminativa/explicativa e a detectar subdimensões cujos conteúdos analisados preliminarmente propiciam avanço na compreensão dos construtos aferidos pelo Inventário.

Palavras-chave: delinquência juvenil; avaliação psicológica; medidas da personalidade; teoria de resposta ao item.

\section{ABSTRACT - Development of the Reduced Brazilian Version of the Jesness Inventory - Revised}

The aim of this study was to select items for the composition of the reduced Brazilian version of the Jesness Inventory - Revised, a specific instrument for the psychological evaluation of juvenile offenders. Data from 954 adolescents of the population aged between 11 and 18 years were used. Analyses using the Item Response Theory Exploratory Model (IRTEM) and then the Item Response Theory Confirmation Model (IRTCM) were performed for each scale of the instrument. In the IRTEM stage, items with an unacceptable $\alpha$ were excluded and in the IRTCM stage only items with moderate or high $\alpha$ values were maintained. Subdimensions were found in the scales. This led to a version with 86 items. The strategies adopted allowed the selection of items with greater discriminative/explanatory capacity and the identification of subdimensions through preliminary content analysis helped in the comprehension of the construct.

Keywords: juvenile delinquency; psychological assessment; personality measurement; item response theory.

\section{RESUMEN - Desarrollo de la Versión Reduzida del Inventário de Jesness - Revisado Brasileiro}

El objetivo de este estudio fue seleccionar ítems para la composición de la versión reducida del Inventario de Jesness - Revisado Brasileiro, una herramienta específica para evaluación psicológica de adolescentes infractores, con una muestra de 954 adolescentes de la población, del sexo masculino y con edades comprendidas entre 11 y 19 años. Para cada escala del instrumento fueron realizadas los Análisis de Modelo Exploratorio de la Teoría de Respuesta al Ítem (EMIRT) y, a continuación, del Modelo Confirmatorio de la Teoría de Respuesta al Ítem (CMIRT). En la etapa del EMIRT se excluyeron ítems con $\alpha$ inaceptable y, en el CMIRT, se mantuvieron sólo ítems con $\alpha$ moderado o alto. Se demostró la existencia de subdimensiones en las escalas, siendo que en algunos ítems se mantuvieron en más de una escala. De esta forma, se obtuvo una versión con 86 ítems. Las estrategias adoptadas permitieron seleccionar ítems con mayor capacidad discriminatoria/explicativa y a detectar subdimensiones cuyos contenidos fueron analizados preliminarmente otorgan avances en la comprensión de los constructos evaluados por el Inventario.

Palabras clave: delincuencia juvenil; evaluación psicológica; medidas de la personalidad; teoría de respuesta al ítem.

No contexto sociocultural brasileiro, ser jovem e pertencer aos estratos socioeconômicos desfavorecidos constitui condição de vulnerabilidade social, especialmente no que diz respeito à violência. Seja em função do arranjo pouco estruturado de suas atividades de rotina, seja em função de algumas de suas características físicas - sexo masculino e cor negra, por exemplo -, o segmento é alvo de maior controle social ostensivo/repressivo e, com isso, experimenta inúmeras violações de direitos (Adorno, 2002). Nessa esteira, muitos são vítimas de violência (inclusive a fatal) (Instituto de Pesquisa Econômica Aplicada [Ipea] \& Fórum Brasileiro de Segurança Pública [FBSP], 2018) e alguns autores de violência, sendo, por isso, apreendidos e processados no âmbito do Sistema de Justiça Juvenil (Brasil, 2018; Instituto Sou da Paz, 2018). 
Os adolescentes que ganham o rótulo de infrator da lei constituem um subgrupo ainda mais vulnerável. Em diferentes realidades sociais, as evidências mostram que a judicialização dos adolescentes tem efeitos negativos significativos, diretos e indiretos, no seu desenvolvimento - sobretudo quando as sanções que recebem envolvem privação de liberdade (Barnert et al., 2017). O estigma, por exemplo, diminui/dificulta o acesso a oportunidades de socialização (Bernburg \& Krohn, 2006), funcionando como fator de risco para a evasão escolar e para a própria manutenção da conduta delituosa (Aizer \& Doyle, 2015). Por essa razão, normativas internacionais (Assembleia Geral das Nações Unidas [AGNU], 1985) e nacionais (Brasil, 1990; Lei n. 12594, 2012) propõem a aplicação racional das sanções aos jovens infratores, com o emprego cauteloso das medidas judiciais. Seja no plano das tomadas de decisão, seja no da execução das sanções, as normativas indicam a importância de considerar as circunstâncias nas quais se deu a prática do delito pelo adolescente, assim como de conhecer suas necessidades desenvolvimentais, no sentido de oferecer-lhe acompanhamento ajustado às suas características psicossociais (Conselho Nacional de Justiça [CNJ] \& Instituto de Pesquisa Econômica Aplicada [Ipea], 2011).

Entende-se que conhecer os adolescentes infratores em suas necessidades desenvolvimentais remete a ações visando compreendê-los naquelas demandas gerais, partilhadas com qualquer outro adolescente de sua faixa de idade, mas também naquelas específicas, atreladas a sua prática de delitos (Casey, 2011). O processo de compreensão implica no de avaliação, para o qual é fundamental obter informações relevantes em torno de variáveis sociais e pessoais-psicológicas, que digam respeito às necessidades desenvolvimentais dos adolescentes. No tocante às necessidades de natureza pessoal, ou mais especificamente psicológica, defende-se que a avaliação é uma tarefa profissional, própria da Psicologia, que requer adoção de procedimentos sistemáticos, incluindo instrumentos padronizados, que resultem em informações úteis e confiáveis (Urbina, 2006).

Sobre o tema, Manzi-Oliveira e Bazon (2013) realizaram uma revisão de literatura científica acerca das principais práticas e recomendações no campo. As autoras identificaram que as principais dimensões focalizadas nos estudos relativos à avaliação psicológica de adolescentes infratores são inteligência, aspectos de saúde mental e características do funcionamento psicológico ou da personalidade, dado que cobrem os aspectos mais frequentemente assinalados nas demandas que o Direito faz à Psicologia, nessa área. No que concerne especificamente à avaliação do funcionamento psicológico ou da personalidade do adolescente infrator, pode-se dizer que esta é norteada pelo objetivo de aceder ao funcionamento psíquico do sujeito, às esferas cognitivas e afetivas, permitindo apreender sua subjetividade e identificar possíveis problemas comportamentais, afetivos ou cognitivos, e potencialidades e capacidades pessoais (Simões, 1999). Em geral, procura atender questionamentos relacionados à sociabilidade do adolescente, sua orientação social, diante da possibilidade de reincidir na prática de delitos e da (re)inserção social.

No Brasil, no âmbito científico, não se denota uma linha clara de investimento nessa temática (ManziOliveira \& Bazon, 2013). Na prática, por seu turno, a necessidade e a importância da avaliação psicológica são reconhecidas nesse campo, embora se observe absoluta falta de consenso sobre o que exatamente avaliar e como fazê-lo, denotando-se ampla variação de critérios e de formas de avaliar os adolescentes em conflito com a lei (Manzi-Oliveira \& Bazon, 2013; Maruschi \& Bazon, 2013). Verifica-se uma tendência à realização de avaliações em uma perspectiva prioritariamente psicopatológica, geralmente orientada pelo conceito de "psicopatia", alinhada a uma visão maniqueísta, prevalente no Direito Criminal, esquecendo-se que a adolescência é uma etapa transitória da vida (Castro \& Guareschi, 2008; Costa, Penso, Sudbrack \& Jacobina, 2011).

No âmbito internacional, por outro lado, a avaliação de adolescentes infratores constitui objeto de interesse acadêmico-científico há muitos anos. Com isso, as pesquisas avançaram para desenvolver ferramentas de avaliação ancoradas em modelos teóricos específicos ao tema, visando instrumentalizar os profissionais e colaborar para a compreensão do fenômeno. Denota-se o esforço para que as avaliações estejam centradas em aspectos notadamente relevantes com relação à prática infracional na adolescência (Le Blanc, 2001). Para além da avaliação do risco de reincidência, baseada em instrumentos atuariais consolidados, bastante conhecidos e difundidos, denotam-se esforços para estabelecer procedimentos sistemáticos voltados ao conhecimento dos adolescentes em suas motivações - crenças, valores e atitudes - retratando aspectos da subjetividade construídos ao longo do tempo, nas interações entre o indivíduo e o contexto, em uma determinada realidade comunitária e sociocultural (Gallo, 2008). O Jesness Inventory - Revised (JI-R; Jesness, 2003) é um instrumento elaborado e aperfeiçoado como um recurso para esse tipo de avaliação.

O JI-R é um dos instrumentos mais amplamente utilizado no contexto do Sistema de Justiça Juvenil Norte americano (EUA e Canadá) para avaliar adolescentes infratores, ao lado do Minnesota Multiphasic Personality Inventory - Adolescent - MMPI-A e do Millon Adolescent Clinical Inventory - MACI (Pinsoneault \& Ezzo, 2011). O JI-R é muito valorizado quando a demanda é compreender o funcionamento psicológico de adolescentes infratores em função de suas especificidades (Wenger \& Pueyo, 2016). Sua aplicação e correção são simples, sendo composto por um conjunto de itens $(n=160)$ de fácil compreensão aos adolescentes. Focaliza variáveis consideradas dinâmicas, atinentes a aspectos do funcionamento psicológico relacionados às condutas antissociais, 
suscetíveis às intervenções psicossociais, como crenças/ valores e atitudes antissociais, e traços de personalidade que possuem relação com condutas de risco, especialmente a impulsividade, a hostilidade e a busca de sensações (Jesness, 2003).

Por essas razões o JI-R caracteriza-se como instrumento muito adequado ao contexto de acompanhamento socioeducativo (Semel, 2016). Mostra-se sensível às mudanças que podem se processar nas cognições, emoções e sentimentos, sejam em função da idade ou de novas experiências, característica que reforça seu potencial de uso nas etapas do acompanhamento socioeducativo/ intervenção psicossocial. Oferece um indicador global, denominado Índice de Associabilidade (IA), aferido a partir da combinação de escores obtidos em algumas de suas escalas, que se relaciona com níveis de risco de reincidência em atos antissociais/delitos, tomando por base a avaliação da orientação (anti)social do adolescente. Avalia ainda outros aspectos do funcionamento psicológico não associados à conduta antissocial/delituosa - pensamentos e sentimentos -, a fim de auxiliar na apreensão da subjetividade do adolescente avaliado, de modo mais geral.

Esse instrumento é estudado na realidade brasileira há algum tempo, de modo que se tem a versão nacional: o Inventário de Jesness - Revisado Brasileiro (IJ-R-Br; Bazon, 2016; Manzi-Oliveira, 2012;). Nos estudos já realizados se obteve evidências de adequadas qualidades psicométricas no IJ-R-Br. Índices de confiabilidade satisfatórios foram encontrados para a maior parte das escalas do instrumento. No que se refere à validade, várias de suas medidas diferenciaram infratores de não infratores de forma bastante precisa (Costa, Komatsu, \& Bazon, 2017; Costa, Komatsu, Manzi-Oliveira, \& Bazon, 2019). Mediante a necessidade de se dispor de instrumentos padronizados, no Brasil, para a área forense, específicos à avaliação de adolescentes infratores, propõe-se aqui a dar continuidade aos estudos sobre o IJ-R-Br. Em efeito, o presente estudo descreve uma das etapas de obtenção de uma versão reduzida do IJ-R-Br, baseada na seleção de itens, usando o modelo multidimensional da Teoria de Resposta ao Item (TRI), ajustado a itens aos quais as respostas podem avaliar múltiplas habilidades/traços (DeMars, 2016).

A proposta de redução do IJ-R-Br associa-se a questões práticas e a questões técnicas. No tocante a questões práticas, embora o Inventário não tenha um número excessivo de itens $(n=160)$, quando comparado a outros instrumentos de avaliação de personalidade tradicionais como, por exemplo, o MMPI-A, ele se destina a um grupo mais específico: adolescentes em conflito com a lei, o que justifica esforços nessa direção. Os adolescentes em conflito com a lei, grosso modo, apresentam características sociais e pessoais mediante as quais um instrumento de avaliação longo pode ser contraproducente para se obter informações com a qualidade almejada. Eles apresentam, em geral, significativa defasagem escolar, em razão de reprovações e/ou de períodos de evasão escolar (Silva, Cianflone, \& Bazon, 2016). Essa condição indica menor proficiência em leitura e interpretação de textos. Assim, quanto mais longo o instrumento proposto a esse grupo, maior o desafio imposto e o risco de se obter respostas baseadas em uma compreensão falha do "texto".

Ademais, Worthington e Whittaker (2006) apontam, tendo por base a literatura especializada em avaliação psicológica, que a aplicação de um instrumento não deve levar mais que 50 minutos e que, a depender do objetivo da avaliação e de quem são os respondentes - sua motivação com respeito à avaliação -, esse tempo deve ser menor e girar entre 15 e 30 minutos. O tempo médio requerido para a aplicação do IJ, conforme consta em seu Manual, é de 20 a 30 minutos (Jesness, 2003). Nas pesquisas realizadas no contexto nacional, esse tem girado em torno dos 30-40 minutos quando autoaplicado e 35-45 minutos quando aplicado na forma de entrevista estruturada, o que ultrapassa em muito o tempo preconizado, sobretudo ao se avaliar adolescentes que, em razão de sua conduta, encontram-se involuntariamente em um contexto regulado por instituições de controle social, circunstância que afeta sua disposição para colaborar com o processo avaliativo.

Em termos técnicos, malgrado as evidências de validade discriminante do IJ-R-Br, sua análise fatorial confirmatória indicou resultados sofríveis em boa parte das escalas, muito provavelmente em função de efeitos de multicolineariedade. Segundo Bazon (2016), mesmo usando o software MPLus, indicado para esse tipo de análise com instrumento com respostas dicotômicas, não foi possível avaliar o modelo completo do IJ-R-Br, possivelmente em função de muitos itens do instrumento comporem duas ou mais escalas simultaneamente. Os índices de ajustamento obtidos para cada uma das escalas em separado ficaram no limite do aceitável, identificando-se itens com cargas fatoriais negativas ou baixas. Como exemplo, a análise da escala denominada Retraimento (Rt) do IJ-R-Br, composta por 22 itens no modelo original, indicou que 18 deles apresentaram carga fatorial negativa, três cargas positivas, porém baixas, e somente um item atingiu índice satisfatório, mesmo diante da relevância clínica dessa escala e das boas evidências de sua validade convergente (Semel, 2016).

Assim, defende-se que a proposição de uma versão reduzida do IJ-R-Br pode proporcionar seu aperfeiçoamento, favorecendo suas propriedades psicométricas (Goetz et al., 2013), e otimizando sua aplicabilidade junto à população específica a qual se destina (Worthington \& Whittaker, 2006). Para tal, reitera-se, recorreu-se à Teoria de Resposta ao Item (TRI), que tem tido o seu uso ampliado na área de redução no número de itens de instrumentos de avaliação, uma vez que estima de forma bastante robusta os itens mais relacionados com o construto de interesse (Edelen \& Reeve, 2007). 


\section{Método}

\section{Participantes}

Os dados utilizados no estudo foram coletados junto a uma amostra de conveniência, composta por 954 adolescentes do sexo masculino ${ }^{2}$, com idades entre 11 e 19 anos, sendo $618(64,8 \%)$ provenientes de escolas do ensino público e $336(35,2 \%)$ de ensino privado do interior do Estado de São Paulo e de Minas Gerais. A Tabela 1 sintetiza a distribuição das idades dos adolescentes da amostra.

Tabela 1

Distribuição da Faixa Etária da Amostra (n=954)

\begin{tabular}{|c|c|c|}
\hline Idade (em anos) & $n$ & $\%$ \\
\hline 11 & 75 & 7,9 \\
\hline 12 & 144 & 15,1 \\
\hline 13 & 133 & 13,9 \\
\hline 14 & 120 & 12,6 \\
\hline 15 & 132 & 13,8 \\
\hline 16 & 161 & 16,9 \\
\hline 17 & 136 & 14,3 \\
\hline 18 & 43 & 4,5 \\
\hline 19 & 10 & 1,0 \\
\hline Total & 954 & 100,0 \\
\hline
\end{tabular}

\section{Instrumentos}

Inventário de Jesness - Revisado - Brasileiro (IJ-R-Br). A coleta de dados foi feita com a aplicação do Inventário de Jesness - Revisado - Brasileiro (IJ-R-Br). Como em sua versão original, em inglês (Jesness, 2003), o IJ-R-Br é composto por 160 itens que o adolescente responde dicotomicamente: Verdadeiro (V) ou Falso (F). Diferentes combinações desses itens resultam em escores em 10 escalas de personalidade: Desadaptação Social (DS); Orientação de Valores (OV); Imaturidade (Im); Autismo (Au); Alienação (Al); Agressividade manifesta (AM); Retraimento/Depressão (Rt/Dep); Ansiedade Social (AS); Repressão (Rep); Negação (Ng). Há também duas escalas que remetem a indicadores comportamentais relacionados a diagnósticos psiquiátricos previstos no DSM-IV (APA, 1994 apud Jesness, 2003): Transtorno de Conduta (TC) e Transtorno Opositivo Desafiador (TOD).

\section{Procedimentos}

Antes de se proceder à coleta de dados, o projeto foi analisado e aprovado pelo Comitê de Ética em Pesquisa com Seres Humanos (CAAE no 86860718.1.0000.5407). $\mathrm{Na}$ sequência, escolas públicas e privadas, que tinham turmas do fundamental II, a partir do $7^{\circ}$ ano até o ensino médio, envolvendo os três anos, em cidades do interior de São Paulo e Minas Gerais (Ribeirão Preto e Juiz de Fora, respectivamente), foram contatadas. Nas escolas, procedeu-se ao convite aos alunos e ao envio de solicitação dos consentimentos dos pais/responsáveis. Junto aos jovens voluntários, autorizados pelos pais/responsáveis, aplicou-se o IJ-R-Br, em pequenos grupos, em salas de aula das próprias escolas.

\section{Análise de Dados}

As análises foram realizadas pelo pacote Multidimensional Item Response Theory (MIRT; Chalmers, 2012) do software R, o qual proporciona parâmetros que descrevem a interação destes com os traços latentes (dimensões/construtos psicológicos medidos) e respostas aos itens. Neste trabalho, o software utilizado não comportou igualmente a realização da análise fatorial confirmatória do modelo completo do IJ-R-Br. A opção foi adotar a estratégia de avaliar cada escala em separado, com uma etapa adicional anterior: Modelo Exploratório de Teoria de Resposta ao Item (EMIRT) para cada uma delas, também em separado, devido a multidimensionalidade inerente às escalas do IJ-R-Br. Nessa etapa, foram gerados modelos de 1 a 7 dimensões para cada escala e comparados por meio da análise de variância. Foram selecionados os melhores modelos, com base nos critérios de informação Bayesiano (BIC) e Akaike (AIC), identificando-se mais de uma subdimensão em todas as escalas, à exceção da escala TC - Transtorno de Conduta e TOD - Transtorno Opositivo Desafiador, que se mantiveram com uma única dimensão.

Após identificar o número de subdimensões em cada escala, os itens que contribuíram pouco para os achados

2 Os estudos de padronização e investigação de propriedades psicométricas devem ser realizados em função do sexo dos respondentes (Jesness, 2003). 
foram excluídos, com base na análise do parâmetro $\alpha$ (índice discriminativo), calculado para cada item, em cada subdimensão da escala. Os valores foram analisados de acordo com parâmetros estabelecidos por Baker (2001): índices entre 0 ou valores inferiores não oferecem discriminação; entre 0,01 e 0,34 oferecem discriminação muito baixa; entre 0,35 a 0,64 oferecem discriminação baixa; entre 0,65 e 1,34 discriminação moderada; e entre 1,35 e 1,69 , alta discriminação. A partir do resultado das análises fatoriais exploratórias (AFEs), com as subdimensões correspondentes a cada escala e um conjunto total menor de itens, passou-se ao Modelo Confirmatório de Teoria de Resposta ao Item (CMIRT), também tratando cada escala separadamente, a fim de avaliar o desempenho dos itens e, então, selecionar aqueles com melhor capacidade discriminativa.

Após esse procedimento foi realizada análise de conteúdo das escalas e suas subdimensões com a intenção de verificar se o significado das escalas da versão reduzida se assemelhava ao da versão original. Para isso, dois juízes com formação em Psicologia e familiarizados com o instrumento avaliaram, de forma independente, a qual construto latente os itens remetiam.
A partir disso analisou-se o construto latente de cada subdimensão e de cada escala, comparando-se a análise independente dos juízes. Em casos de discordância, um terceiro juiz foi consultado para a tomada da decisão técnica sobre o item.

\section{Resultados}

Os resultados das EMIRT para cada escala em separado apontaram para soluções de três a quatro subdimensões em cada escala do instrumento - à exceção das indicativas de transtornos, que se mostraram unifatoriais, conforme já mencionado. Nessa primeira etapa de seleção de itens, foram excluídos itens cujos índices discriminativos se mostraram menores que 0,34 - considerando que não ofereciam discriminação ou que esta era muito baixa (Baker, 2001). A Tabela 2 resume a informação sobre o número/a quantidade de itens segundo a classificação $\alpha$, por escala. À frente do nome de cada escala há, entre parêntesis, o número total de itens que compõe cada escala, no instrumento original, e na coluna seguinte o número de subdimensões identificadas inicialmente em cada escala correspondente.

Tabela 2

Número de Itens por Escala Conforme a Classificação dos $\alpha$, a Partir da EMIRT

\begin{tabular}{|c|c|c|c|c|c|}
\hline \multirow[b]{2}{*}{$\begin{array}{c}\text { Escala } \\
\left(\mathrm{n}^{\circ} \text { inicial de itens) }\right.\end{array}$} & \multirow[b]{2}{*}{$\begin{array}{l}\text { Número de } \\
\text { dimensões }\end{array}$} & \multirow[b]{2}{*}{$\begin{array}{c}\text { Itens } \\
\text { excluídos } \\
(\alpha \text { até } 0,34)\end{array}$} & \multicolumn{3}{|c|}{ Itens retidos e seu $\alpha$} \\
\hline & & & $\begin{array}{c}\text { Baixa } \\
(\alpha \text { entre } \\
0,35 \text { e } 0,64)\end{array}$ & $\begin{array}{c}\text { Moderada } \\
\text { ( } \alpha \text { entre } \\
0,65 \text { e } 1,34)\end{array}$ & $\begin{array}{c}\text { Alta } \\
(\alpha>1,35)\end{array}$ \\
\hline 1. Desadaptação Social (62) & 3 & 14 & 10 & 35 & 3 \\
\hline 2. Orientação de Valores (38) & 4 & 29 & 5 & 2 & 2 \\
\hline 3. Imaturidade (29) & 4 & 5 & 14 & 5 & 5 \\
\hline 4. Autismo (26) & 4 & 7 & 8 & 8 & 3 \\
\hline 5. Alienação (25) & 4 & 5 & 7 & 7 & 6 \\
\hline 6. Agressividade Manifesta (31) & 4 & 2 & 7 & 15 & 7 \\
\hline 7. Retraimento (22) & 4 & 3 & 3 & 9 & 7 \\
\hline 8. Ansiedade Social (20) & 4 & 3 & 6 & 9 & 2 \\
\hline 9. Negação (19) & 4 & 5 & 6 & 8 & - \\
\hline 10. Repressão (14) & 4 & 1 & 3 & 8 & 2 \\
\hline 11. TC (14) & 1 & 1 & 0 & 8 & 5 \\
\hline 12. TOD (16) & 1 & 2 & 1 & 9 & 4 \\
\hline
\end{tabular}

Por vezes, um mesmo item foi retirado de uma escala, mas preservado em outra, quando nesta apresentou desempenho adequado ( $\alpha$ satisfatório). Os procedimentos de seleção de itens não objetivaram tornar os itens exclusivos a cada escala. Todavia, houve, com tal procedimento, uma melhor depuração nestes. Os números nas colunas referentes às faixas de discriminação "Baixa", "Moderada" e "Alta" do $\alpha$ indicam a quantidade de itens em cada uma, por escala. A partir desse resultado, ou seja, mantendo-se nas escalas os itens com valores $\alpha$ nessas faixas, passou-se à etapa da CMIRT. Destaca-se que em algumas escalas, o mesmo item compõe mais de uma subdimensão identificada. Por exemplo, na escala denominada Imaturidade (Im), o item 55 compõe duas das quatro subdimensões, tendo em ambas $\alpha$ na classe "Alta". Assim, esse item foi contabilizado só uma vez. Contudo, nos casos em que o mesmo item apresentou valores $\alpha$ em diferentes faixas, para fins de apresentação de dados, manteve-se a regra de contabilizar cada item uma só vez, privilegiando o registro do melhor desempenho do item. 
Após a exclusão de itens, algumas subdimensões identificadas a priori foram suprimidas, especificamente nas escalas Retraimento (Rt), Negação (Neg), Agressividade Manifesta (AM), Alienação (Al) e Orientação de Valores $(\mathrm{OV})$. Na Tabela 3, nota-se um número inferior de subdimensões identificadas em relação ao resultado da EMIRT. Isso ocorreu porque todos os itens de determinadas subdimensões situaram-se na faixa de "não discriminação" ou "muito baixa discriminação", o que resultou em modelos mais simples para estas escalas.

Tabela 3

Classificação dos $\alpha$ dos Itens para Cada Escala a Partir da CMIRT

\begin{tabular}{|c|c|c|c|c|}
\hline \multirow[b]{2}{*}{$\begin{array}{c}\text { Escala } \\
\left(\mathrm{n}^{\circ} \text { final itens) }\right.\end{array}$} & \multirow[b]{2}{*}{$\begin{array}{l}\text { Número de } \\
\text { dimensões }\end{array}$} & \multirow[b]{2}{*}{$\begin{array}{l}\text { Itens excluídos } \\
(\alpha \text { até } 0,34)\end{array}$} & \multicolumn{2}{|c|}{ Itens retidos } \\
\hline & & & $\begin{array}{c}\text { Moderada } \\
\text { ( } \alpha \text { entre } 0,65 \text { e } \\
1,34)\end{array}$ & $\begin{array}{c}\text { Alta } \\
(\alpha>1,35)\end{array}$ \\
\hline 1. Desadaptação Social (41) & 3 & 9 & 26 & 15 \\
\hline 2. Orientação de Valores (15) & 3 & 6 & 8 & 7 \\
\hline 3. Imaturidade (9) & 4 & 15 & 4 & 5 \\
\hline 4. Autismo (11) & 4 & 8 & 6 & 5 \\
\hline 5. Alienação (8) & 3 & 12 & 4 & 4 \\
\hline 6. Agressividade Manifesta (14) & 3 & 15 & 10 & 4 \\
\hline 7. Retraimento (6) & 1 & 13 & 3 & 3 \\
\hline 8. Ansiedade Social (9) & 4 & 8 & 7 & 2 \\
\hline 9. Negação (8) & 2 & 6 & 5 & 3 \\
\hline 10. Repressão (7) & 4 & 6 & 3 & 4 \\
\hline 11. TC (9) & 1 & 4 & 3 & 6 \\
\hline 12. TOD (5) & 1 & 9 & 2 & 3 \\
\hline
\end{tabular}

Nessa etapa, a lógica foi selecionar itens com melhor desempenho, e não excluir aqueles com desempenho insatisfatório. Dessa forma, mantiveram-se itens somente com $\alpha$ moderado e alto. Os seguintes itens (86 ao total) foram selecionados para compor a versão reduzida do instrumento: $3 ; 4 ; 10 ; 11 ; 14 ; 15 ; 17 ; 19 ; 20 ; 22 ; 23 ; 25$; 27 ; $28 ; 30 ; 31 ; 33 ; 35 ; 36 ; 37 ; 44 ; 49 ; 51 ; 55 ; 58 ; 60 ; 61$; $63 ; 64 ; 65 ; 66 ; 67 ; 68 ; 69 ; 71 ; 72 ; 73 ; 76 ; 77 ; 78 ; 79 ; 83$; $87 ; 88 ; 91 ; 93 ; 94 ; 95 ; 96 ; 98 ; 99 ; 101 ; 102 ; 103 ; 104 ; 107$; $111 ; 112 ; 113 ; 115 ; 117 ; 121 ; 122 ; 123 ; 125 ; 128 ; 129$; $130 ; 131 ; 133 ; 135 ; 138 ; 140 ; 142 ; 143 ; 144 ; 145 ; 146$; $147 ; 148 ; 150 ; 152 ; 153 ; 154 ; 157 ; 159$.

Quando comparados os dados das tabelas 2 e 3, percebe-se que, após exclusão de itens, para a maioria das escalas, o número bruto de itens com valor $\alpha$ considerado alto, aumentou ou se manteve igual, mesmo com a diminuição do número total de itens. Como o procedimento foi realizado por escala, alguns dos itens que permaneceram no instrumento deixaram de pertencer a algumas escalas. Por exemplo, o item 111, "Em casa, muitas vezes eu sou culpado por coisas que eu não fiz", originalmente compartilhado por cinco escalas, na proposta de versão reduzida, passou a compor três. O item 147, "Para o meu tamanho, eu sou um cara durão", originalmente pertencente a quatro escalas, na versão reduzida, foi mantido somente em duas.

A Tabela 4 apresenta a síntese da análise preliminar de conteúdo das escalas e das subdimensões identificadas. Foi verificado que o significado teórico das escalas foi mantido. Alguns conteúdos se repetem em subdimensões de diferentes escalas, por exemplo, o construto "Desconfiança das figuras de autoridade" aparece em duas subdimensões de DS e em uma subdimensão de OV. Assim ocorre com o conteúdo "Atitude favorável a comportamentos antissociais" que aparece em duas subdimensões de DS, em uma subdimensão de OV e em uma subdimensão de Au.

Tabela 4

Análise Preliminar de Conteúdo das Escalas e Subdimensões do Instrumento Final

\begin{tabular}{clcl}
\hline Escala & \multicolumn{1}{c}{ Definição } & $\begin{array}{c}\text { Número } \\
\text { Itens }\end{array}$ & \multicolumn{1}{c}{ Subdimensões - Descrição } \\
\hline DS & 22 & $\begin{array}{l}\text { Desconfiança das figuras de autoridade; Atitude favorável a } \\
\text { resolução de conflitos por meio da força física; Orientação } \\
\text { antissocial }\end{array}$ \\
\cline { 2 - 3 } & $\begin{array}{l}\text { a adoção de } \\
\text { antissociais }\end{array}$ & 25 & $\begin{array}{l}\text { Desconfiança das figuras de autoridade; Sentimento de mal-estar e } \\
\text { inadequação social; Orientação antissocial }\end{array}$ \\
\cline { 2 - 3 } & 3 & Autoconceito positivo \\
\hline
\end{tabular}


Tabela 4 (continuação)

Análise Preliminar de Conteúdo das Escalas e Subdimensões do Instrumento Final

\begin{tabular}{|c|c|c|c|}
\hline Escala & Definição & $\begin{array}{l}\text { Número } \\
\text { Itens }\end{array}$ & Subdimensões - Descrição \\
\hline \multirow{3}{*}{ OV } & \multirow{3}{*}{$\begin{array}{l}\text { Crenças simplistas } \\
\text { sobre a realidade }\end{array}$} & 8 & Atitude favorável a comportamentos antissociais e fatalismo \\
\hline & & 4 & Percepção de relações conflituosas na família \\
\hline & & 4 & Desconfiança das figuras de autoridade \\
\hline \multirow{4}{*}{$\operatorname{Im}$} & \multirow{4}{*}{$\begin{array}{l}\text { Pensamentos comuns } \\
\text { a pessoas mais novas }\end{array}$} & 1 & Busca por sensação/estimulação \\
\hline & & 4 & Lócus de controle externo \\
\hline & & 3 & Dificuldade em controlar o próprio comportamento \\
\hline & & 3 & Ingenuidade na avaliação de situações/motivações \\
\hline \multirow{3}{*}{$\mathrm{Au}$} & \multirow{3}{*}{$\begin{array}{l}\text { Distorção da percepção } \\
\text { de si e da realidade } \\
\text { em benefício próprio }\end{array}$} & 8 & Distorção da realidade, percepção de desconforto e solidão \\
\hline & & 3 & Atitude favorável a comportamentos antissociais \\
\hline & & 3 & Autoestima inflada \\
\hline \multirow{3}{*}{$\mathrm{Al}$} & \multirow{3}{*}{$\begin{array}{l}\text { Estranhamento } \\
\text { face ao outro }\end{array}$} & 6 & Atitude negativa/ fatalismo \\
\hline & & 4 & Desconfiança dos outros e desesperança \\
\hline & & 3 & Atitude negativa/ fatalismo \\
\hline \multirow{3}{*}{$\mathrm{AM}$} & \multirow{3}{*}{$\begin{array}{l}\text { Percepção de } \\
\text { sentimentos de raiva }\end{array}$} & 13 & Sensação de que não ser capaz de controlar emoções e ações \\
\hline & & 11 & Percepção de sentimentos de raiva \\
\hline & & 5 & Percepção de sentimentos negativos \\
\hline Rt & $\begin{array}{l}\text { Emotividade } \\
\text { negativa / desamparo }\end{array}$ & 6 & Emotividade negativa/desamparo \\
\hline \multirow{4}{*}{ AS } & \multirow{4}{*}{$\begin{array}{l}\text { Sentimentos de } \\
\text { ansiedade em } \\
\text { situações sociais }\end{array}$} & 4 & Ansiedade geral \\
\hline & & 1 & Autoestima frágil \\
\hline & & 2 & Ansiedade em relação à própria performance \\
\hline & & 4 & Ansiedade em interações sociais \\
\hline \multirow{2}{*}{ Neg } & \multirow{2}{*}{$\begin{array}{l}\text { Negação aspectos } \\
\text { negativos na família } \\
\text { e em si }\end{array}$} & 2 & Negação de aspectos negativos na família \\
\hline & & 7 & Negação de aspectos negativos na família e em si \\
\hline \multirow{4}{*}{ Rep } & \multirow{4}{*}{$\begin{array}{l}\text { Repressão aspectos } \\
\text { negativos individuais e } \\
\text { relacionais }\end{array}$} & 3 & Repressão de aspectos negativos em si e nas relações \\
\hline & & 4 & Repressão de aspectos negativos relacionais \\
\hline & & 5 & Repressão de aspectos negativos em si e nas relações \\
\hline & & 3 & Autoestima inflada (superestimação das capacidades) \\
\hline
\end{tabular}

\section{Discussão}

De forma geral, em comparação aos resultados descritos por Bazon (2016), os achados do presente estudo mostraram que os modelos e os itens do IJ-R-Br tiveram um desempenho melhor, com índices de ajustamento adequado e índices discriminativos mais diversificados. Acredita-se que isso se deve à técnica utilizada nas análises, a Teoria de Resposta ao Item (TRI). Por mais que tenham resultados análogos, a Teoria Clássica de Testes (TCT) e a TRI implicam em procedimentos distintos, sendo que para instrumentos em que se detecta multidimensionalidade, a TRI é mais apropriada, ao passo que para instrumentos de estruturas unidimensionais, a TCT e a TRI se equivalem (Raykov \& Marcoulides,
2016). O melhor desempenho dos itens também pode estar associado à identificação das subdimensões, resultantes das EMIRT. A apreensão de multidimensionalidade nas escalas do IJ-R-Br pode significar um importante avanço na compreensão do inventário e na sua melhor exploração, uma vez que sinalizam questões fundamentais da estrutura interna do instrumento. $\mathrm{O}$ arranjo em subdimensões permite avaliar o desempenho dos itens de uma nova maneira, agrupados em subconjuntos com mais alta relação com um construto/traço latente aferido. Como exemplo, retomam-se os resultados da escala Rt, cujo desempenho foi insatisfatório nas análises realizadas por Bazon (2016), a despeito de evidências sólidas de validade convergente (Semel, 2016), o que não é incomum. No presente, os resultados da EMIRT apontaram, 
inicialmente, quatro subdimensões, as quais, após exclusão de itens insatisfatórios e realização de CMIRT, resultaram em uma dimensão, com seis itens com capacidade discriminativa moderada e alta, o que retrata um aperfeiçoamento substancial da escala. Assim, parece que tratar Rt como unifatorial/unidimensional, quando, na verdade, possuía quatro fatores/dimensões, prejudicava o desempenho dos itens nas avaliações.

A hipótese que emerge dos resultados aqui obtidos é de que isso ocorreu em praticamente todas as escalas do IJ-R-Br, no estudo anterior (Bazon, 2016), em maior ou menor grau. Para Rt, após a seleção de itens, três subdimensões da escala foram suprimidas, tornando a estrutura mais simples, assim como para três outras do Inventário.

Ainda que o formato ideal das escalas de um instrumento de avaliação psicológica seja a unifatorial (em termos estatísticos), as evidências encontradas não permitiram alcançar essa condição. Ademais, os procedimentos de seleção de itens não objetivaram alcançar a exclusividade dos itens nas escalas. Para tal, seria necessário avaliações de conteúdo dos itens - semelhante àquelas realizadas por ocasião da elaboração do instrumento correndo-se o risco de desperdiçar o potencial teórico inerente à multidimensionalidade do próprio do inventário, tão válida clinicamente. De todo modo, a exclusão de itens pelos procedimentos adotados concorreu para a diminuição no número de itens compartilhados entre as escalas, assim como simplificou a estrutura de algumas delas. Todavia, o fato de alguns itens terem se mantido em mais de uma escala simultaneamente sugere que estes são multidimensionais em relação aos traços/construtos que medem.

A seleção dos 86 itens, por meio de procedimentos estritamente estatísticos, é uma limitação. A análise de seus conteúdos é importante. Entretanto, essa decisão foi tomada com base em um método de análise de dados mais avançado (TRI), o qual ofereceu a segurança de uma avaliação mais sofisticada do inventário, com resultados que ajudaram a selecionar os itens que melhor discriminaram adolescentes com alto nível no construto medido em relação aos demais. Ainda, defende-se que a estratégia para classificação dos índices discriminativos foi ponderada, visto que não foram selecionados somente itens com alto desempenho, para que se evitasse excluir itens potencialmente relevantes em termos teóricos.
Obviamente, os resultados têm de ser tomados com cautela, dado que a intenção foi não alterar a estrutura do instrumento, mas compreendê-la melhor a fim de decidir as futuras etapas de investigação que possam tornar o IJ-R-Br viável para uso profissional. Um dos esforços nesse sentido será utilizar as subdimensões identificadas para aprimorar a descrição das escalas do IJ-R-Br ou até mesmo verificar se não houve modificações significativas em sua capacidade interpretativa, por meio da avaliação de conteúdo por especialistas, ainda que na análise preliminar de conteúdo aqui realizada se considerou que as escalas mantiveram os significados teóricos do instrumento original.

De todo modo, considera-se que tenham sido excluídos itens com pouca relação com o construto medido. Nesse sentido, análises relativas a evidências de validade convergente das diferentes medidas da versão reduzida do inventário são oportunas. No momento, trabalha-se na coleta de dados com a aplicação dessa versão reduzida em amostra independente para verificar as propriedades psicométricas deste conjunto de itens remanescentes.

O desenvolvimento de ferramentas de avaliação psicológica específicas para a área forense, concernindo especificamente à problemática relativa ao envolvimento de adolescentes com a prática de atos infracionais, como o IJ-R-Br, em paralelo a outros avanços na avaliação de aspectos relevantes nas esferas familiar, escolar e comunitária, representa um passo importante à superação de um cenário de práticas pouco sistemáticas, no Brasil, no âmbito dos programas e serviços relativos ao Sistema de Justiça Juvenil (Maruschi \& Bazon, 2013). Representa um recurso alinhado ao que preconiza a legislação internacional e nacional, referente à necessidade de entender o adolescente para além do ato infracional que praticou (Estatuto da Criança e do Adolescente [ECA], 1990; Conselho Nacional os Direitos da Criança e do Adolescente [CONANDA] \& Secretaria do Desenvolvimento Humano [SEDH], 2002; Lei n.12594, 2012), mas também contornando o viés de vê-lo tão somente pela perspectiva da psicopatologia (Gallo, 2008). A possibilidade de usar adequadamente instrumento como o Inventário de Jesness na área forense ajudará a deixar para trás a tendência de compreender o adolescente pelo prisma do delito cometido, o que, ao longo da história, vem concorrendo para práticas tutelares, baseadas em disciplina punitiva (Rodrigues, Oliveira, \& Rocha, 2017).

\section{Referência}

Adorno, S. (2002). Youth crime in São Paulo: Myths, images and facts. Em S. Rotker (Ed.), Citizens of fear - Urban violence in Latin America (pp. 102-116). New York: Rutgers, The State University of New Jersey Press.

Aizer, A., \& Doyle, J. J. (2015). Juvenile incarceration, human capital and future crime: Evidence from randomly assigned judges. The quartely journal of economics, 130(2), 759-803. doi: 10.1093/qje/qjv003 
Assembleia Geral das Nações Unidas. (1985). Regras de Beijing.

Baker, F. (2001). The basics of item response theory. College Park, MD: Eric Clearinghouse on Assessment and Evaluation.

Barnert, E. S., Dudovitz, R., Nelson, B. B., Coker, T. R., Biely, C., Li, N., \& Chung, P. J. (2017). How does incarcerating young people affect their adult health outcomes? Pediatrics, 139(2). doi: 10.1542/peds.2016-2624

Bernburg, J. G., \& Krohn, M. D. (2006). Labeling, life chances and adult crime: The direct and indirect effects of official intervention in adolescence on crime in early adulthood. Criminology, 41(4), 1287-1318. doi: 10.1111/j.1745-9125.2003.tb01020.x

Bazon, M. R. (2016). Avaliação Psicológica de Adolescentes em Conflito com a Lei: Validação do Inventário de Jesness - Revisado (Tese de Livre Docência). Universidade de São Paulo, Ribeirão Preto, São Paulo.

Brasil (1990). Estatuto da Criança e do Adolescente: Lei Federal 8.069/90. Belo Horizonte: CEDCA-MG, 136p

Brasil (2018). Levantamento anual SINASE 2016. Brasília: Ministério dos Direitos Humanos. Brasília,DF: MDH.

Casey, S. (2011). Understanding young offenders: Developmental criminology. The Open Criminology Journal, 4, 13-22. doi: $10.2174 / 1874917801104010013$

Castro, A. L. S., \& Guareschi, P. (2008). Da provação da dignidade social à provação da liberdade individual. Psicologia \& Sociedade, 20(2), 200-207.

Costa, L. F., Penso, M. A., Sudbrack, M. F. O., \& Jacobina, M. P. (2011). Adolescente em conflito com a lei: O relatório psicossocial como ferramenta para promoção do desenvolvimento. Psicologia em Estudo, 16(3), 379-387.

Chalmers, R. P. (2012). Mirt: A multidimensional item responsa theory package for The R environment. Jornal of Statistical Software, 48(6). doi: $10.18637 /$ jss.v048.i06

Conselho Nacional os Direitos da Criança e do Adolescente \& Secretaria do Desenvolvimento Humano (2002). Sistema Nacional de Atendimento Socioeducativo - SINASE. Secretaria Especial dos Direitos Humanos, Brasília: CONANDA.

Conselho Nacional de Justiça \& Instituto de Pesquisa Econômica Aplicada (2011). Justiça infanto-juvenil: Situação atual e critérios de aprimoramento. Relatório de Pesquisa. Brasília, DF: CNJ \& Ipea.

Costa, R., Komatsu, A. V., \& Bazon, M. R. (2017). Psychological assessment of adolescent offenders. International Annals of Criminology, 55(1), 60-77. doi:10.1017/cri.2017.2

Costa, R. C. S., Komatsu, A. V., Manzi-Oliveira, A. B., \& Bazon, M. R. (2019). Avaliação psicológica em delinquência juvenil: Confiabilidade e de validade do Inventário de Jesness, Psico PUC RS, 3(50). (no prelo).

DeMars, C. R. (2016) Partially compensatory multidimensional item Response Theory Models: Two alternate model forms. Educational and Psychological Measurement, 76(2), 231-257. doi: 10.1177/0013164415589595

Estatuto da Criança e do Adolescente, Lei no 8069 de 13 de julho de 1990. Brasília: Secretaria Especial dos Direitos Humanos da Presidência da República. Subsecretaria de Promoção dos Direitos da Criança e do Adolescente.

Edelen, M. O., \& Reeve, B. B. (2007) Applying item response theory (IRT) modeling to questionnaire development, evaluation, and refinement. Quality of Life Research: An International Journal of Quality of Life Aspects of Treatment, Care and Rehabilitation, 16(1), 5-18. doi: 10.1007/s11136-007-9198-0

Gallo, A. E. (2008) Atuação do Psicólogo com adolescentes em conflito com a lei: A Experiência do Canadá. Psicologia em Estudo, 13(2), 327334, doi: 10.1590/S1413-73722008000200015

Goetz, C., Coste, J. , Lemetayer, F., Rata, A., Montel, S., Recchia, S., Debouverie, M., Pouchot, J., Spitz, E., \& Guillemin, F. (2013) Item reduction based on rigorous methodological guidelines is necessary to maintain validity when shortening composite measurement scales. Journal of Clinical Epidemiology 66, 710-718. doi: 10.1016/j.jclinepi.2012.12.015

Instituto Sou da Paz (2018). Aí eu voltei para o corre: Estudo da reincidência infracional do adolescente no Estado de São Paulo. São Paulo (SP): Instituto Sou da Paz. Recuperado de http://www.soudapaz.org/upload/pdf/ai_eu_voltei_pro_corre_2018.pdf

Instituto de Pesquisa Econômica Aplicada \& Fórum Brasileiro de Segurança Pública. (2018). Atlas da Violência 2018. Rio de Janeiro (RJ): Ipea e FBSP.

Jesness, C. F. (2003). Jesness Inventory-Revised. Technical Manual. North Tonawanda, USA: MHS.

Le Blanc, M. (2001). MASPAQ: Mesures de L'adaptation Sociale et Personnelle pour les Adolescents Québécois. Manuel et Guide D'utilisation (4a ed.). Montréal: Université de Montréal.

Lei n. 12.594, 18 de janeiro de 2012 (2012). Institui o Sistema Nacional de Atendimento Socioeducativo - SINASE, regulamenta a execução da medida socioeducativa e altera algumas leis. Brasília, DF: SINASE.

Maruschi, M. C., \& Bazon, M. R. (2013). Justiça juvenil: A aplicação e a execução das medidas socioeducativas pelos parâmetros do modelo "Risco-Necessidade- Responsividade". In Prêmio Innovare: 10 Anos - A Justiça do Século XXI, (1), pp. 42-72. Rio de Janeiro: Instituto Innovare.

Manzi-Oliveira, A. B. (2012). Avaliação de adolescentes em conflito com a lei: adaptação transcultural do Inventário de Personalidade de Jesness (Dissertação de mestrado). Faculdade de Filosofia, Ciências e Letras de Ribeirão Preto, Universidade de São Paulo, Ribeirão Preto.

Manzi-Oliveira, A. B., \& Bazon, M. R. (2013) Avaliação psicológica de adolescentes infratores: Uma revisão sistemática da literatura nos últimos dez anos. Em Roberto da Silva, João Clemente de Souza Neto e Francisca Rodrigues Pini (Eds.). Ciência da delinquência: O olhar da USP sobre o ato infracional, o infrator, as medidas socioeducativas e suas instituições. São Paulo (SP): Expressão e Arte.

Pinsoneault, T. B., \& Ezzo, F. R. (2011). Efficacy of the Jesness Inventory-Revised Conduct Disorder and Oppositional Defiant Disorder Scales. Journal of Knowledge and Best Practices in Juvenile Justice and Psychology, 5(1), 31-36.

Raykov, T., \& Marcoulides, G. A. (2016) On the relationship between classical Test Theory and Item Response Theory: From one to the other and back. Educational and Psychological Measurement, 76(2) 325-338, doi: 10.1177/0013164415576958

Rodrigues, C. D., Oliveira, V. C., \& Rocha, R. L. S. (2017) Brazil. Em S. H. Decker \& N. Marteache (2017). International Handbook of Juvenile Justice (pp. 71-89). USA: Ed. Springer. doi: 10.1007/978-3-319-45090-2.

Semel, R. A. (2016) Incorporating the Jesness Inventory-Revised (JI-R) in a best-practice model of juvenile delinquency assessments. Journal of Forensic Psychology Practice, 16(1), 1-23. doi.: 10.1080/15228932.2016.1119516

Silva, J. L., Cianflone, A. R. L., \& Bazon, M. R. (2016) School bonding of adolescent offenders. Paidéia (Ribeirão Preto), 26, 91-100. doi: 10.1590/1982-43272663201611.

Simões, M. R. (1999). O ensino e a aprendizagem da avaliação psicológica: O caso da avaliação da personalidade. Psychologica, 22, 135-172.

Urbina, S. (2006) Fundamentos da testagem psicológica. Porto Alegre (RS): Artmed. 
Wenger, L., \& Pueyo, A. (2016). Tests forenses en español para evaluar adolescentes infractores. Papeles del Psicólogo, 37(2), 107-117.

Worthington, R. L., \& Whittaker, T. A. (2006). Scale development research a content analysis and recommendations for best practices. The Counseling Psychologist, 34, 806-838. doi: 10.1177/0011000006288127

\section{Sobre os autores}

Rafaelle C. S. Costa é psicóloga e mestranda em Psicologia na Faculdade de Filosofia Ciências e Letras da Universidade de São Paulo. André Vilela Komatsu é psicólogo e doutor em Psicologia pela Faculdade de Filosofia Ciências e Letras da Universidade de São Paulo. Lais Sette Galinari é psicóloga e mestre em Psicologia pela Faculdade de Filosofia Ciências e Letras da Universidade de São Paulo.

Marina Rezende Bazon é psicóloga e professora associada do Departamento de Psicologia da Faculdade de Filosofia Ciências e Letras de Ribeirão Preto, Universidade de São Paulo. 